\title{
Tunable Graphene Phononic Crystal
}

\author{
Jan N. Kirchhof,* Kristina Weinel, Sebastian Heeg, Victor Deinhart, Sviatoslav Kovalchuk, Katja Höflich, \\ and Kirill I. Bolotin*
}

Cite This: Nano Lett. 2021, 21, 2174-2182

Read Online

ACCESS | W W Metrics \& More | 国 Article Recommendations | st Supporting Information

ABSTRACT: In the field of phononics, periodic patterning controls vibrations and thereby the flow of heat and sound in matter. Bandgaps arising in such phononic crystals (PnCs) realize low-dissipation vibrational modes and enable applications toward mechanical qubits, efficient waveguides, and state-of-the-art sensing. Here, we combine phononics and two-dimensional materials and explore tuning of PnCs via applied mechanical pressure. To this end, we fabricate the thinnest possible $\mathrm{PnC}$ from monolayer graphene and simulate its vibrational properties. We find a bandgap in the megahertz regime within which we localize a defect mode with a small effective mass of $0.72 \mathrm{ag}=0.002 \mathrm{~m}_{\text {physical }}$. We exploit graphene's flexibility and simulate mechanical tuning of a finite size
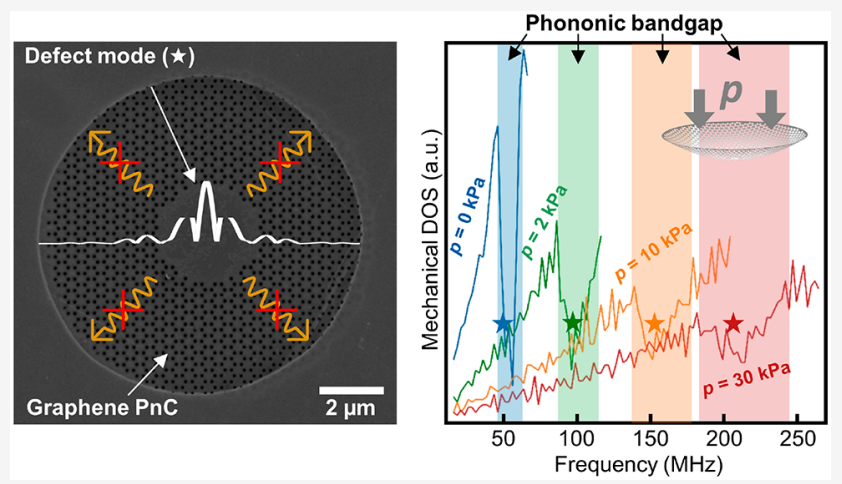
PnC. Under electrostatic pressure up to $30 \mathrm{kPa}$, we observe an upshift in frequency of the entire phononic system by $\sim 350 \%$. At the same time, the defect mode stays within the bandgap and remains localized, suggesting a high-quality, dynamically tunable mechanical system.

KEYWORDS: Nanomechanics, phononic crystal, graphene, optomechanics, resonators, NEMS

\section{INTRODUCTION}

A phononic crystal (PnC) is an artificially manufactured structure with a periodic variation of material properties, for example, stiffness, mass, or stress. ${ }^{1}$ This periodic perturbation creates a meta-crystallographic order in the system leading to a vibrational band structure hosting acoustic Bloch waves in analogy to the electronic band structure in solids. ${ }^{1}$ Designing the lattice parameters of the meta-structure allows one to directly manipulate phonons at various length scales. ${ }^{2-4}$ This can be used to guide $\mathrm{e}^{5-7}$ and focus phonons ${ }^{8,9}$ or to open a vibrational bandgap. ${ }^{1,10-12}$

Phononic bandgaps in periodic structures suppress radiation losses and allow for highly localized modes (of frequency $f$ ) on artificial irregularities. ${ }^{13,14}$ The quality factors $\left(Q=\frac{f}{\Delta f}\right)$ of these so-called defect modes are especially high. ${ }^{15,16}$ In particular, resonances with $Q>8 \times 10^{8}$ have been observed at room temperature in silicon nitride $(\mathrm{SiN}) \mathrm{PnCs}^{15-17}$ In these devices, the quality factor exceeds the empirical $Q \sim m^{1 / 3}$ rule, ${ }^{17-19}$ and the vibrational periods overcome the thermal decoherence time limit of $\tau=h Q / k_{\mathrm{B}} T .^{15,17}$ This, in turn, enables the study of quantum effects in resonators of macroscopic size, all at room temperature. ${ }^{20,21}$

Frequency tunability in PnCs could add an unprecedented knob to control a broad range of phononic application and thereby provides access to new regimes of guiding, filtering, and focusing phonons. ${ }^{22-33}$ It would furthermore allow one to resonantly couple to an external optical or mechanical excitation and thus realize sensing applications with mechanical qubits and studies on quantum entanglement. ${ }^{34}$ Yet, the mechanical resonances in $\mathrm{PnCs}$ are determined by material constants and the crystal geometry. ${ }^{22,23,26-28}$ In principle, the mode frequencies can be controlled by changing the temperature $^{29,30}$ or by an external magnetic field. ${ }^{31,32}$ This, however, only provides limited tunability and necessitates heating the system or inclusion of magnetic materials. While $\mathrm{SiN}$, as well as other conventional low-loss materials, is very stiff and allows only limited mechanical tunability, ${ }^{24,33}$ strain has been used to adjust the frequency response of elastic polydimethylsiloxane (PDMS). ${ }^{25}$ Unfortunately, low crystalline quality of that material led to limited tunability and very small $Q$ s for mechanical modes.

Recently, PnCs made from two-dimensional (2D) materials have been considered. ${ }^{35-37}$ Such materials feature intrinsically low mass, high fundamental frequency, and easily accessible displacement nonlinearity. Most importantly, their high tensile

Received: December 18, 2020

Revised: February 7, 2021

Published: February 23, 2021 
A

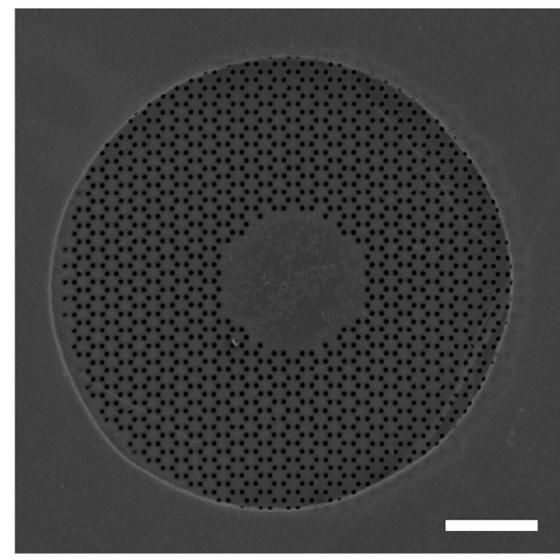

B

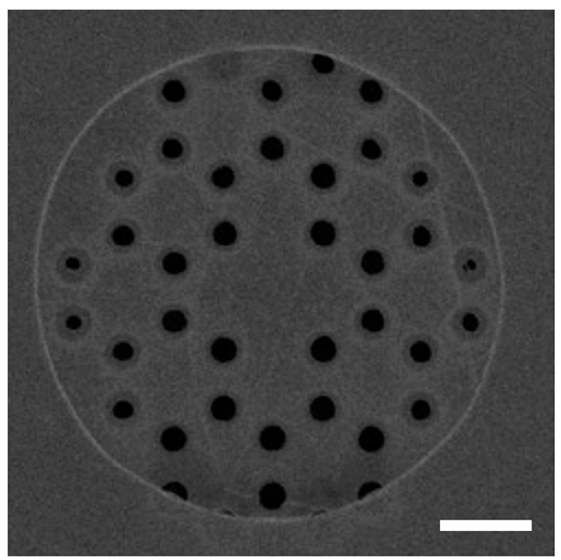

\section{C}

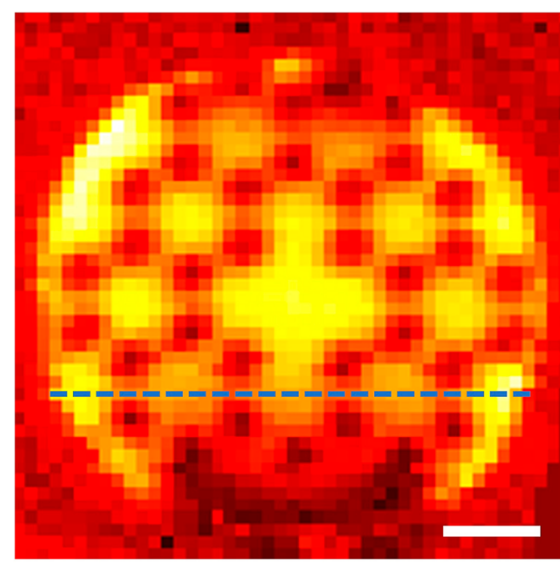

$\mathrm{I}_{2 \mathrm{D}}$ (a.u.)

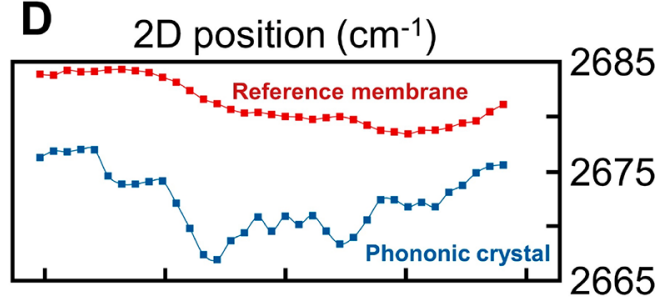

0.5

E Relative tension $(\mathrm{N} / \mathrm{m})$

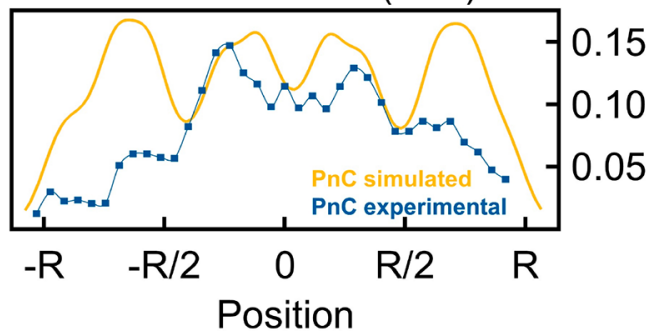

Figure 1. Graphene phononic crystals and tension redistribution. (A,B) Helium ion micrographs of prototype monolayer graphene phononic crystal devices with lattice constants $350 \mathrm{~nm}$ and $2 \mu \mathrm{m}$, respectively. Scale bar length is $2 \mu \mathrm{m}$. The phononic pattern, a honeycomb lattice of holes with a defect in its center, allows us to localize a vibrational defect mode. The ringlike features around the holes in (B) are due to incomplete removal of graphene most likely caused by contamination (details in Supporting Information). (C) Intensity map of the Raman-active 2D mode of graphene for the device shown in (B). The periodic pattern is clearly visible. (D) Raman 2D-mode position along a line cut (dashed line in (C)) for a PnC (blue) and reference membrane (red). The PnC shows a periodic variations of much larger amplitude compared to the fluctuation in the reference sample. (E) Comparison of the relative tension extracted from Raman measurements (blue) to the simulated tension distribution (yellow) confirming the redistribution of tension upon pattering. The simulation includes spatial broadening due to the finite size of the laser spot.

strength and monolayer character allows the ability to mechanically strain them up to $10 \% .{ }^{38}$ That invites consideration of mechanically controllable $2 \mathrm{D}$-material based PnCs. Specifically, we expect the entire acoustic band structure of such a PnC to be highly tunable by applying mechanical pressure. Nevertheless, tunability of $2 \mathrm{D}$ phononic systems as well as localized defect modes in them have not been studied yet.

Here, we investigate mechanical tunability in a realistic graphene PnC. We fabricate a suspended micron-sized monolayer graphene $\mathrm{PnC}$ via focused helium ion beam milling (FIB) and characterize it spectroscopically. We then use experimentally established parameters to calculate the phononic band structure of the resulting PnC. We find a phononic bandgap from 48.8 to $56.5 \mathrm{MHz}$ inside of which we localize a defect mode with an effective mass of 0.72 ag. Finally, we computationally investigate the mechanical tunability of the
PnC under pressure induced by a local electrostatic gate. ${ }^{39,40}$ The applied pressure smears out the phononic bandgap as the out-of-plane displacement breaks the symmetry and causes perturbations of the artificial lattice, yet the mode shape of the defect mode remains highly localized. Overall, we can tune the resonance frequency of the defect mode by more than $350 \%$ and access new regimes of strain engineering.

\section{RESULTS}

Designing a Tunable Phononic Crystal. Our device design of a tunable, two-dimensional $\mathrm{PnC}$ consists of the following key elements. First, the $\mathrm{PnC}$ material must be freestanding to allow out-of-plane displacement. Second, it is necessary to use an electrically conductive material. In that case, an electrostatic gate electrode can be used to apply pressure and to induce tension as the membrane is pulled toward the gate. Third, the material needs to be flexible to 

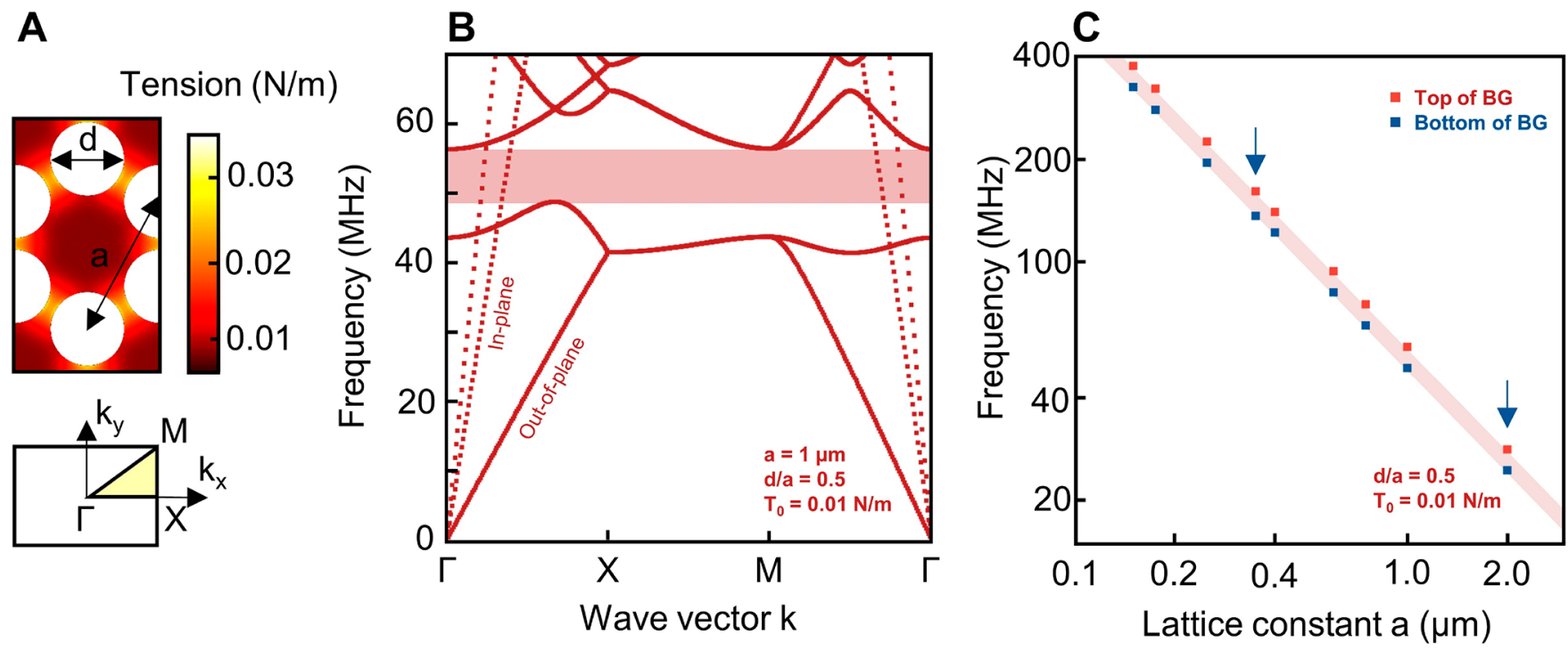

Figure 2. Band structure calculations of an infinite graphene phononic crystal. (A) Unit cell of the honeycomb lattice with redistributed tension (top) and the corresponding first Brillouin zone (bottom). (B) Phononic band structure for the unit cell shown in (A). In-plane modes are shown as dashed lines, out-of-plane modes as solid lines, and the corresponding quasi-bandgap region as the red-shaded area. (C) Top (red) and bottom (blue) of the bandgap versus lattice constant. The blue arrows indicate the lattice constant of the devices from Figure 1.

allow large mechanical tunability with small pressures. Monolayer graphene with its high carrier mobility $>200000$ $\mathrm{cm}^{2} /(\mathrm{V} \mathrm{s})^{41}$ and large breaking strength $>10 \%{ }^{38}$ perfectly fulfils these requirements. By using large area CVD graphene, we can fabricate many devices on a single chip. Finally, the device needs to host a large enough number of unit cells with sufficient periodicity to form a well-defined PnC. While this task is simple in thick $\mathrm{SiN}$, it is much more challenging for fragile freestanding monolayer graphene. To overcome this, we choose a much smaller unit cell compared to typical SiN-PnCs $(\sim 100 \mu \mathrm{m}$ size $)$ and use helium FIB-milling to pattern the $\mathrm{PnC}^{42}$ This direct lithography allows one to pattern graphene down to $10 \mathrm{~nm}$ features, ${ }^{43}$ while causing little damage. ${ }^{44,45} \mathrm{~A}$ patterned prototype monolayer graphene $\mathrm{PnC}$ is shown in Figure 1A. It consists of a honeycomb lattice of holes (lattice constant $a=350 \mathrm{~nm}$, hole diameter $d=105 \mathrm{~nm}$ ) around a central region. Within its $10 \mu \mathrm{m}$ diameter, the two-dimensional $\mathrm{PnC}$ contains more than 30 unit cells. The honeycomb lattice inspired by Tsaturayn et al. ${ }^{15}$ exhibits a robust bandgap ${ }^{12,15,46}$ while retaining a relatively large fraction of material to ensure a stable device. Additional $\mathrm{PnC}$ with various patterning sizes are shown in Figures $\mathrm{S} 1-\mathrm{S} 3$.

Next, we map the tension within the produced structures using Raman spectroscopy. We expect tension hot spots in the thin ribbons and relaxation in the centers of the hexagons. ${ }^{47}$ Such tension redistribution should affect the vibrational properties of our PnC. To this end, we fabricate another prototype device (Figure 1B) with lattice constant $a=2 \mu \mathrm{m}$ and spatial features comparable to the size of a focused laser spot. The intensity map of the 2D-Raman mode of graphene for this device is shown in Figure 1C. The intensity of the 2Dmode corresponds to the amount of material while its spectral position depends on the tension in the material. ${ }^{48,49}$ In the pizza-like image, one can clearly see the removed material from the drop in intensity and identify the honeycomb lattice. In Figure 1D, we compare the spectral position of the Raman 2Dmode for a graphene $\mathrm{PnC}$ (blue) along the dashed line shown in Figure 1C to an unpatterend graphene membrane (red). The quasi-periodic variations in the PnC device that are absent in the unpatterned reference correspond to the redistributed tension. In Figure 1E, we compare the extracted relative tension (blue) to a simulation (yellow) and find the expected signatures of tension redistribution, that is, higher tension between the holes and lower tension in the middle of the hexagons (details in Supporting Information).

Phononic Crystal Simulations. Having experimentally established the feasibility of a suspended graphene $\mathrm{PnC}$, we use our findings to simulate its phononic properties in two independent approaches. First, we calculate the phononic band structure for an infinitely repeated unit cell ("infinite model"). This model is well-accepted and fast. ${ }^{15-17}$ However, due to the size limits of suspended graphene, our devices are smaller than typical SiN-PnCs (mm size) $)^{15-17}$ and contain fewer unit cells. Furthermore, we want to apply pressure to the entire system and investigate localized modes in the bandgap. Therefore, we also simulate a more realistic system of finite size ("finite model"). For both models, we use the honeycomb lattice with feasible parameters and account for tension redistribution upon fabrication (Figure 1D,E). We choose a lattice constant $a$ $=1 \mu \mathrm{m}$, a filling factor of $d / a=0.5$ (slightly larger than in Figure 1), and an initial tension of $T_{0}=0.01 \mathrm{~N} / \mathrm{m}$, which is a realistic value for clean monolayer graphene. ${ }^{39,50}$

Infinite Model. By applying periodic boundary conditions to the unit cell (Figure 2A), we calculate the band structure for an infinite honeycomb lattice (Figure $2 \mathrm{~B}$ ). We find a mixture of in-plane (dashed lines) and out-of-plane modes (solid lines). From the slope of the out-of-plane modes in Figure 2B, we determine the speed of sound $v_{\mathrm{g}}=\frac{\partial \omega}{\partial k}=83 \mathrm{~m} / \mathrm{s}$. In the range from 48.8 to $56.5 \mathrm{MHz}$ (red shaded area), we find a bandgap for out-of-plane modes. This quasi-bandgap (in-plane modes are still present) has a gap-to-midgap ratio of $14.6 \%$. The in-plane modes do not couple to out-of-plane modes ${ }^{51}$ and therefore do not hinder radiation shielding. The bandgap originates from Bragg scattering, with each hole acting as a scatterer for out-of-plane oscillations. Upon negative interference conditions, directional Bragg bandgaps open at the high symmetry points. Where these gaps overlap, radiation shielding 
A

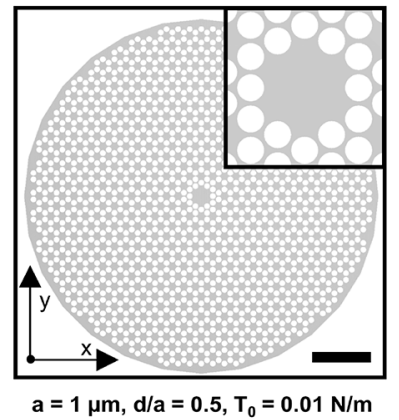

E

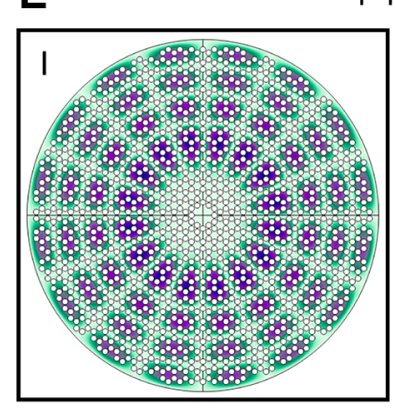

B

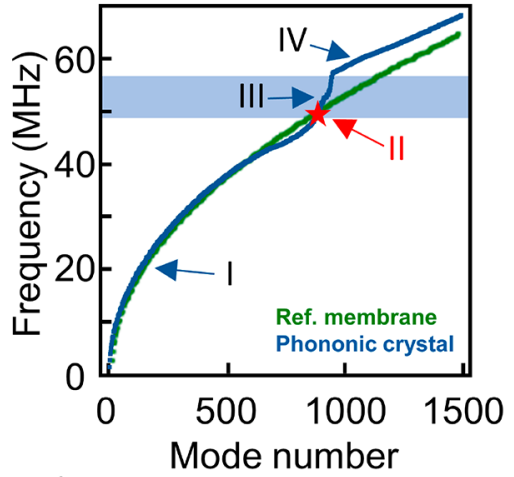

z| (a.u.) $\mathbf{F}$

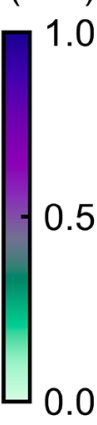

C

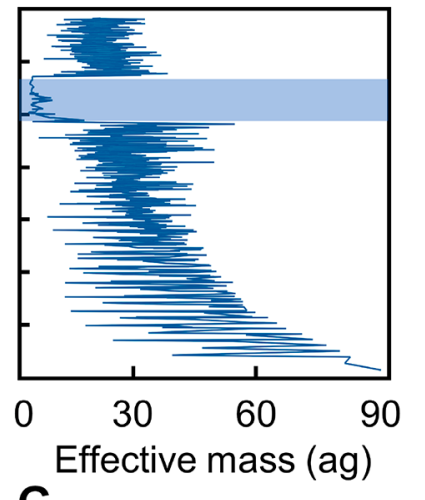

G
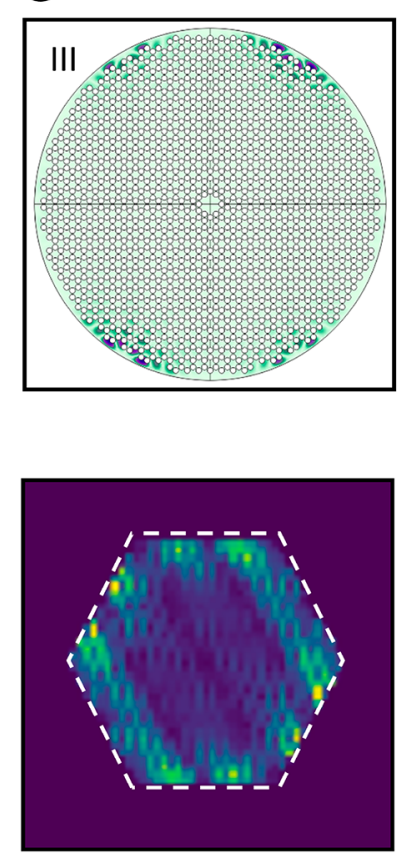

D

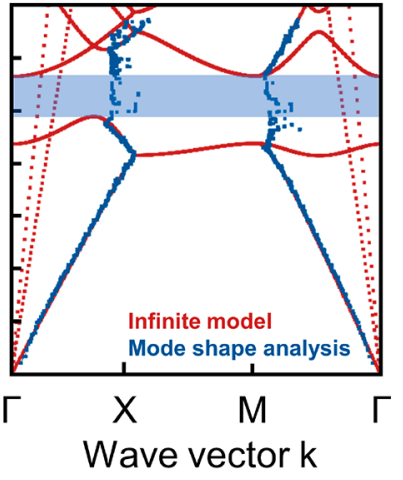

H

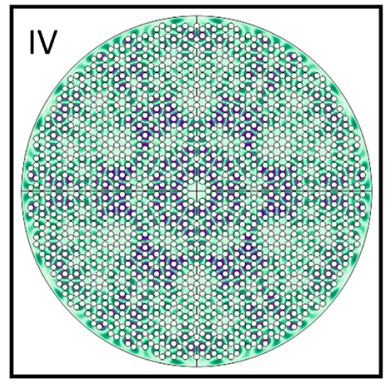

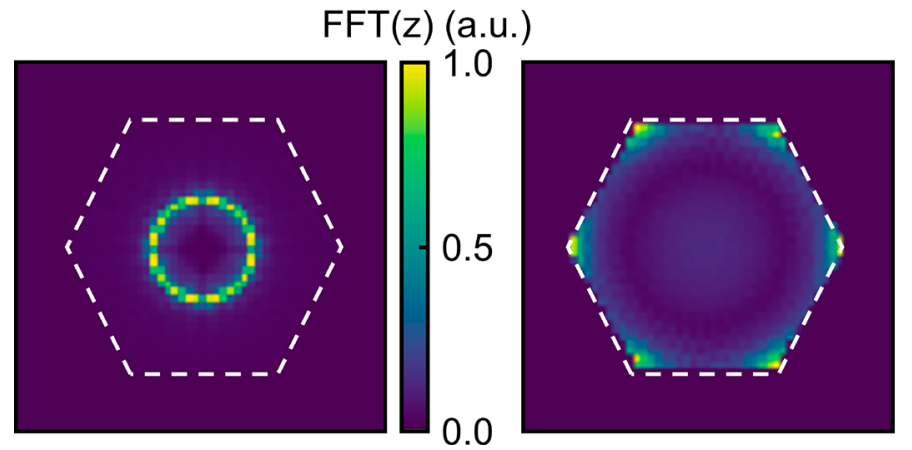

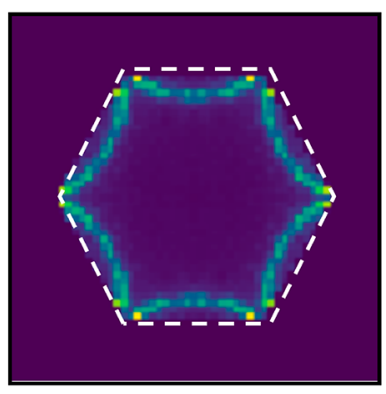

Figure 3. Finite size model of a graphene phononic crystal. (A) Device geometry for the finite system simulations (scale bar is $5 \mu \mathrm{m}$ ). A central "defect" region is designed to localize one vibrational mode and decouple it from its environment. (B) The first 1500 simulated eigenfrequencies versus mode number for a $\mathrm{PnC}$ device (blue) and a circular membrane without patterning (green). The bandgap region from the infinite model is shown in blue. (C) Effective mass for each mode. The modes within the bandgap (blue) show a more than a 100-fold decrease in effective mass compared to the fundamental mode. (D) Band structure calculated from the finite model via mode-shape analysis (blue) along with the band structure from the infinite model (red). The low-energy acoustic branches fit well, and the bandgap regions coincide with the simulated results from the infinite model (red). (E-H) Exemplary mode shapes in real (top) and reciprocal space (bottom) for (E) a mode below the bandgap (I), (F) the defect mode (II), (G) another highly localized mode in the bandgap (III), and (H) a mode above the gap (IV).

becomes possible, as wave propagation is isotropically forbidden. ${ }^{1}$ The bandgap position depends reciprocally on $a$. With our fabrication schema, we can tailor the bandgap center from 350 to $26 \mathrm{MHz}$ by varying $a$ from 0.175 to $2 \mu \mathrm{m}$ (Figure $2 \mathrm{C}$, devices in Figures S2 and S3). Overall, the simulations in the infinite model suggest the possibility of a large quasibandgap, which we will next use to control phonons.

Finite Model. To study a realistic device of finite size under electrostatic pressure and to implement a defect into the phononic pattern, we conduct a second independent simulation ("finite model"). In this model, we consider a finite number of unit cells of the honeycomb lattice (same $a$, $d / a$, and $T_{0}$ as before) and employ fixed boundary conditions along the PnC's perimeter. We choose a circular device as such a geometry allows uniform suspension and minimizes edge effects. In the center of the $30.6 \mu \mathrm{m}$ device, we create a $1.9 \mu \mathrm{m}$ hexagonal defect, ${ }^{15}$ as sketched in Figure 3A. Freestanding graphene devices of that size have been fabricated ${ }^{52}$ and the central defect area is large enough to measure resonances interferometrically. ${ }^{53,54} \mathrm{Next}$, we simulate the first 1500 eigenfrequencies and the corresponding spatial mode shape. In Figure 3B, we plot the frequencies $f$ versus mode number $N$ for the PnC (blue) and compare it to an unpatterned graphene membrane as reference (green). The graph for the PnC shows signs of a bandgap, as we observe an initial flattening of the curve followed by a sudden increase. This region of reduced mode density coincides exactly with the bandgap from our infinite model (blue area) and stands in contrast to the unpatterned membrane for which the frequencies gradually increase with mode number. The second indication of the bandgap is evident when we examine the effective mass of the modes 


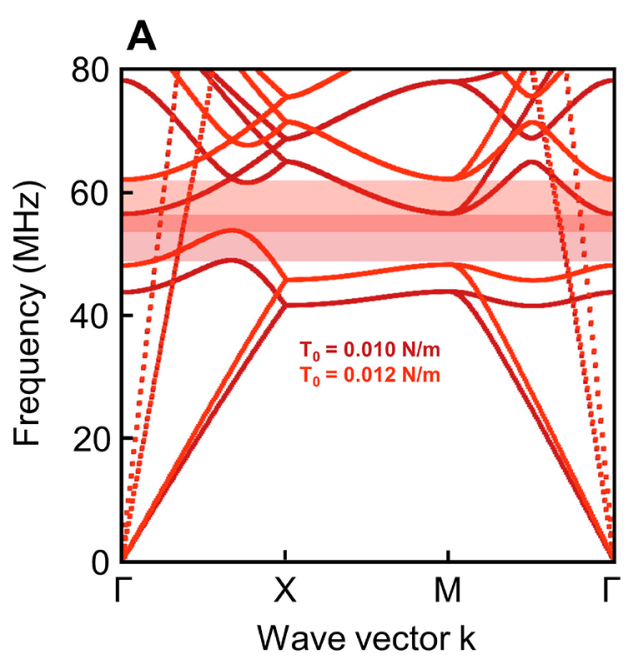

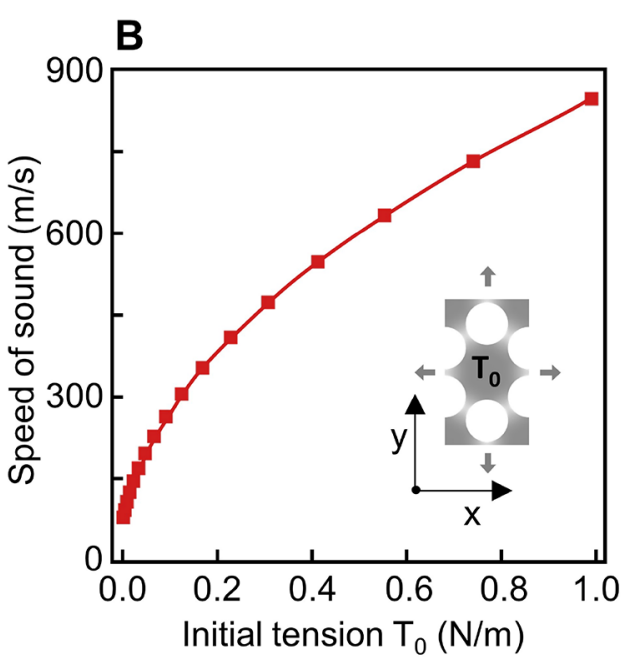
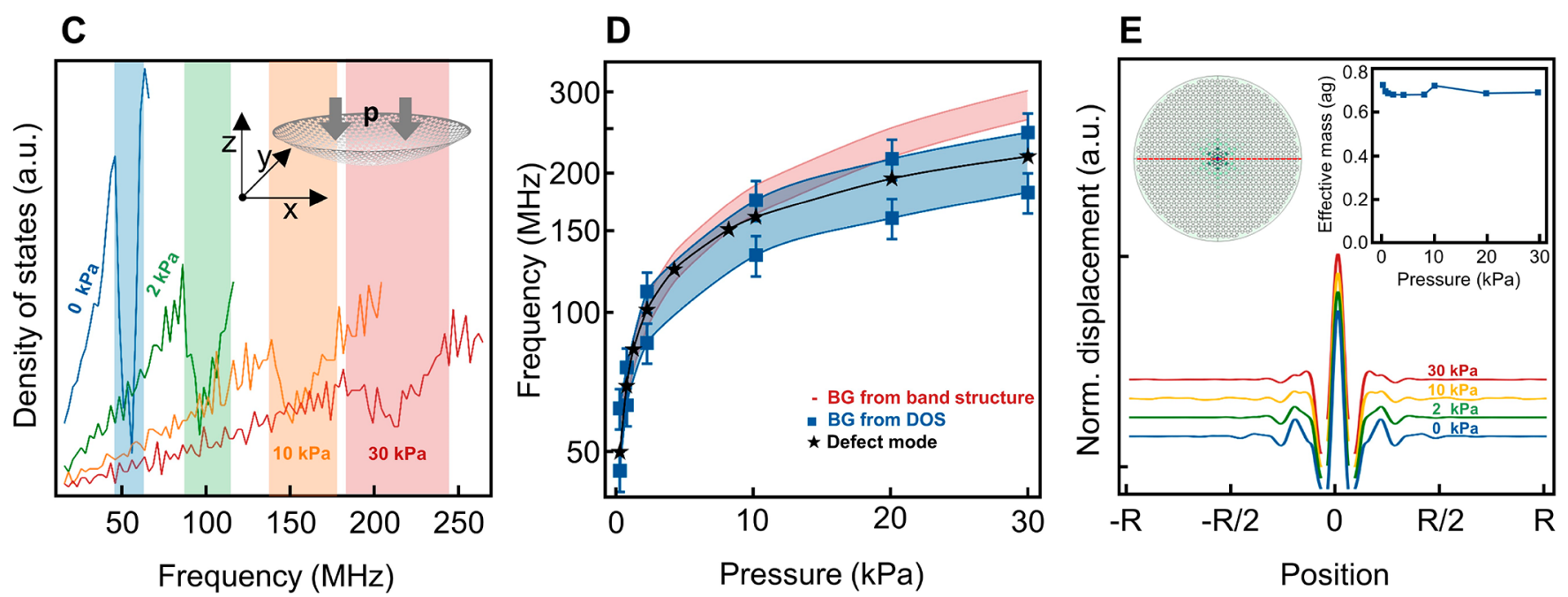

Figure 4. Mechanically tunable graphene phononic crystal. (A) Band structure for initial tension values $T_{0}=0.010 \mathrm{~N} / \mathrm{m}(\mathrm{red})$ and $T_{0}=0.012 \mathrm{~N} / \mathrm{m}$ (orange). The entire out-of-plane branch scales strongly with tension. The position and width of the bandgap are equally tension-dependent. (B) Speed of sound for the out-of-plane modes extracted from (A) versus tension. (C) Density of states calculated from the finite model as a function of pressure applied to the suspended $\mathrm{PnC}\left(T_{0}=0.010 \mathrm{~N} / \mathrm{m}\right)$. (D) Pressure dependence of resonance frequency of the central defect mode (stars), of the bandgap from infinite model (red shaded), and of the bandgap extracted from the density of states (blue squares). The defect mode remains within the bandgap even at high pressures. (E) Line cut for the spatial profile of the defect mode at different pressures (vertically offset for clarity). Even at large applied loads, the mode shape remains localized, and the effective mass (inset) stays constant.

$$
m_{\text {eff }}=\rho_{2 D} \iint \frac{z^{2}}{z_{\max }^{2}} \mathrm{~d} x \mathrm{~d} y
$$

where $\rho_{2 \mathrm{D}}$ is the areal density of graphene and $z\left(z_{\max }\right)$ is the (maximum) vibration amplitude in $z$-direction. For the fundamental mode we obtain $m_{\text {eff }}=80.9 \mathrm{ag}=0.252 \mathrm{~m}_{\text {physical }}$ which roughly matches the literature value for the mode shape of a uniform, circular membrane of $\mathrm{m}_{\text {eff }}=0.269 \mathrm{~m}_{\text {physical }}{ }^{55} \mathrm{We}$ observe a pronounced drop of $m_{\text {eff }}$ in the bandgap region (Figure 3C). This observation is consistent with localized modes inside the bandgap, which typically show a small average displacement resulting in a reduced effective mass. ${ }^{17}$

Finally, we directly extract the band structure from the results of the finite model and compare it to that of the infinite model. To accomplish this, we analyze the mode shape of each resonance following ref 56. Specifically, we take the spatial FFT of each mode shape to find its representation in reciprocal space and to assign a wave vector $k$ to each mode. In Figure $3 \mathrm{E}-\mathrm{H}$, we show real space (top) and reciprocal space (bottom) plots of exemplary modes. Mode I (20.2 MHz, Figure $3 \mathrm{E}$ ) is below the bandgap and resembles a higher order Bessel mode in real space, which transforms to a near-uniform circle in momentum space. A higher frequency mode IV (60.7 $\mathrm{MHz}$, Figure $3 \mathrm{H})$ is situated above the bandgap. For this mode, we observe zone folding as the mode reaches out beyond the 1.BZ (dashed line). Analyzing all 1500 modes lets us restore the dispersion relation beyond the 1.BZ (Figure 3D, blue), which almost perfectly matches the band structure from the infinite model (red). From our observations of reduced mode density (Figure 3B), drop in effective mass (Figure 3C), and mode shape-analysis (Figure 3D), we confirm the presence of a bandgap for out-of-plane modes in a realistic system of finite size.

Next, we examine the modes located within the bandgap and identify the defect mode. In Figure 3G, we show a typical bandgap mode in real (top) and $k$-space (bottom). As most modes in the bandgap, this mode is localized at the edges of the $\mathrm{PnC}$ in the real space. However, one mode at frequency 
49.9 $\mathrm{MHz}$ is localized at the central defect (Figure 3F) and surrounded by the phononic pattern. We therefore identify it as our defect mode of special interest. The $m_{\text {eff }}$ of the mode is $0.724 \mathrm{ag}$, which is more than a factor of 100 smaller than the fundamental mode of the system and orders of magnitude lower than for any reported SiN defect mode. ${ }^{15-17}$ Overall, our model confirms the vibrational bandgap for a system of finite size and a localized defect mode within that bandgap.

Phononic Crystal Tuning. We now show the key advantage of our graphene $\mathrm{PnC}$ : dynamic and rapid frequency tuning of the bandgap and of the defect mode. To demonstrate this, we model our graphene $\mathrm{PnC}$ under pressure, which is applied by an electrostatic gate. The pressure causes displacement of the suspended membrane and increases the in-plane tension. We initially approximate this effect in first order in our infinite model by neglecting out-of-plane displacement and simply increasing the in-plane tension. In Figure 4A, we plot the band structure for $T_{0}=0.010$ and $0.012 \mathrm{~N} / \mathrm{m}$. We observe a frequency increase of the out-of-plane modes and thus an upshift of the quasi-bandgap by $10 \%$. The speed of sound $v_{\mathrm{g}}$ rises from 83 to $830 \mathrm{~m} / \mathrm{s}$ in the range of tension from 0.01 to 1 $\mathrm{N} / \mathrm{m}$ (Figure 4B). The system (finite and infinite) behaves like a thin membrane under tension, and the frequencies of the PnC scale directly with tension: $f \propto \sqrt{\frac{T_{0}}{\rho_{2 \mathrm{D}}}} \cdot 55$ This scaling makes our system highly sensitive to tension and in combination with graphene's mechanical flexibility allows for broad frequency tuning.

Having demonstrated the overall tunability of our system, we now simulate the effect of electrostatic pressure on the phononic system and the defect mode in a realistic device. To do so, we switch to the finite model and apply pressure in negative $z$-direction. In our simulations, we stick to experimentally reported pressure values and apply a maximum of $30 \mathrm{kPa}^{39}$ To investigate the influence of pressure on the bandgap, we compute the phononic density of states, DOS = $\mathrm{d} N / \mathrm{d} f$, and plot it versus pressure (Figure 4C). In this plot, the bandgap is distinguished by a reduced DOS. While at zero pressure the bandgap region is obvious, for higher pressures the drop becomes less pronounced (Figure 4C). We attribute this smearing to a breaking of symmetry, perturbation of the $\mathrm{PnC}$ as it deforms under pressure (inset Figure $4 \mathrm{C}$ ), and rising nonuniformity in the tension distribution (Figure S6E). Nevertheless, we estimate the top and bottom of the bandgap, Figure 4D (blue). A bandgap tuning by more than $300 \%$ is evident. We verify the bandgap tuning by an independent approach based on averaging the induced tension (red markers, details in Supporting Information).

Next, we investigate tunability of the defect mode. Upon applying $30 \mathrm{kPa}$ pressure to a device with an initial tension of $0.01 \mathrm{~N} / \mathrm{m}$, the resonance frequency of the defect mode upshifts from 49.9 to $217.5 \mathrm{MHz}$ (black stars Figure 4D). Because the bandgap is smeared under pressure (Figure 4C), it is important to check the localization of the defect mode. Hence, we inspect a line cut through the center of the device and plot the normalized mode shape versus pressure in Figure 4E. The shape as well as the effective mass (inset Figure 4E) of the mode remains virtually unchanged and the mode retains its localization. Summarizing, we have shown a tunable speed of sound and realized an upshift of the defect mode resonance under pressure, while maintaining its localization. Such a more than 4-fold frequency increase is unprecedented and remains elusive in any other phononic systems. ${ }^{2-33}$

\section{DISCUSSION}

We now discuss experimental signatures of this system. The spatial features of the extended modes in our device (Figure $3 \mathrm{E}, \mathrm{H})$ are too fine to be resolved via diffraction-limited optics. At the same time, the extent of the defect mode is in the size of microns (Figure $3 \mathrm{~F}$ ) allowing the detection of that mode via interferometric read-out (Figure S8). ${ }^{53,54}$ This mode has a nonzero net displacement and can be directly actuated via electrostatic drive. It will be straightforward to distinguish the defect mode from other modes by its localization in the center of the device and its likely increased quality factor. Indeed, the quality factor is defined by $Q=2 \pi E_{\text {stored }} / E_{\text {diss }}$, where $E_{\text {diss }}$ is the dissipated energy per oscillation including all dissipation mechanisms and $E_{\text {stored }}$ is the mode's total energy. As the mode shape shows zero displacement near the clamping points, we expect strongly suppressed bending losses and thus enhanced Q's. Additionally, the phononic shield hinders radiation losses into the substrate, which become especially important at higher frequencies. ${ }^{16}$ While bending and radiation losses may play a secondary role among the mechanisms lowering $Q$ in graphene resonators, our experiments nevertheless should determine the contribution of these mechanisms. Finally, by applying pressure we increase the stiffness of the resonator. This increases the energy stored in the system ${ }^{17}$ and supposedly further enhances the quality factor. The demonstrated level of strain control in our system invites future studies on dissipation dilution via strain engineering following the work of Ghadimi et al. ${ }^{17}$

We also note that our results can be easily extended to the entire family of two-dimensional materials. Currently, it is challenging for us to experimentally achieve sufficient uniformity in the graphene membrane in order to generate a spatially uniform bandgap and localize the defect mode. Monolayer graphene is rather sensitive to surface corrugations ${ }^{39}$ and transferred CVD graphene is often covered by fabrication residues, so using thin exfoliated graphene multilayers could be a solution for which we expect to find experimental signatures. The increased uniformity in multilayer graphene comes along with a decreased tunability, yet we anticipate more than $100 \%$ relative tuning for up to $\sim 35$ layers (Figure S9). For our graphene PnC, we do not expect to reach $Q$ 's comparable to SiN. Nevertheless, we estimate $m_{\text {eff }}$ of our defect mode to be at least 8 orders of magnitude lower than in other 2D-SiN-PnCs. ${ }^{15}$ This immensely increases the measurement rate of quantum states $\Gamma_{\text {meas }} \propto 1 / m_{\text {eff }}$ and decreases thermomechanical noise. ${ }^{15}$ The frequencies in our system are controlled by simply adjusting a gate voltage, and we expect the tuning to take place on time scales comparable to regular graphene resonators and therefore achieve tuning bandwidths $>15 \mathrm{kHz}{ }^{57}$

\section{CONCLUSION}

In summary, we have fabricated and simulated a tunable PnC made from monolayer graphene. For an experimentally informed honeycomb lattice structure, we find a robust vibrational bandgap in the megahertz range. The bandgap persists for a finite-size system, and we use it to localize a defect mode and shield it from its surroundings. This defect mode shows a very small effective mass of $0.724 \mathrm{ag}$, orders of magnitude smaller compared to traditional PnCs. As our central result, we demonstrate a frequency upshift of the defect mode as well as the entire phononic system by more than 
$350 \%$ by applying an experimentally feasible pressure of 30 $\mathrm{kPa}$. While the bandgap smears out due to out-of-plane displacement perturbing the lattice, the defect mode stays within the bandgap and remains highly localized. We suggest experimental signatures of the defect mode allowing its differentiation from other modes in the system. Overall, our design of a 2D-material-based $\mathrm{PnC}$ adds a new knob to dynamically and rapidly tune frequencies in a broad range of phononic applications. Our results invite future experiments as our approach allows adjustable coupling of a PnC to external systems and may lead to better understanding of the dissipation mechanisms in graphene.

\section{METHODS}

Device Fabrication. The pattering of the CVD grown graphene membranes was carried out in a He-ion microscope (Orion Nanofab). Supporting Information Section I provides a detailed process description.

Raman Spectroscopy. Raman mapping was performed on a Horiba Xplora Raman spectrometer using a 100× (NA 0.9) objective and $532 \mathrm{~nm}$ excitation. Spectra were acquired with a laser power of $0.5 \mathrm{~mW}$ and an integration time of $3 \mathrm{~s}$. Tension (via strain) values were derived from the $2 \mathrm{D}$-mode position following standard procedures, see Supporting Information Section IV.

Simulations. For the finite element modeling we use COMSOL Multiphysics (Version 5.5) and assume the following material parameters for monolayer graphene: Young's modulus $E_{2 \mathrm{D}}=1.0 \mathrm{TPa},{ }^{38}$ Poisson's ratio of $v=$ 0.15 , thickness of $h=0.335 \mathrm{~nm}$, and a density of $\rho=\frac{\rho_{2 \mathrm{D}}}{h}=$ $2260 \mathrm{~kg} / \mathrm{m}^{3}$. The initial tension $T_{0}=0.01 \mathrm{~N} / \mathrm{m}$ thus corresponds to an initial strain: $\epsilon_{0}=\frac{T_{0}}{E_{2 \mathrm{D}}} \approx 0.003 \%$. For details see Supporting Information, Sections II and III.

\section{ASSOCIATED CONTENT}

\section{(s) Supporting Information}

The Supporting Information is available free of charge at https://pubs.acs.org/doi/10.1021/acs.nanolett.0c04986.

Graphene pattering using He-FIB milling, details of the FEM-simulations and the mode shape analysis, detailed Raman spectroscopy analysis, and simulations on experimental signatures (PDF)

\section{AUTHOR INFORMATION}

\section{Corresponding Authors}

Jan N. Kirchhof - Department of Physics, Freie Universität Berlin, 14195 Berlin, Germany; 이이이.org/0000-00018576-4787; Email: jan.kirchhof@fu-berlin.de

Kirill I. Bolotin - Department of Physics, Freie Universität Berlin, 14195 Berlin, Germany; Email: kirill.bolotin@fuberlin.de

\section{Authors}

Kristina Weinel - Department of Physics, Freie Universität Berlin, 14195 Berlin, Germany; Ferdinand-Braun-Institut gGmbH Leibniz-Institut für Höchstfrequenztechnik, 12489 Berlin, Germany

Sebastian Heeg - Department of Physics, Freie Universität Berlin, 14195 Berlin, Germany; (1) orcid.org/0000-00026485-3083
Victor Deinhart - Ferdinand-Braun-Institut gGmbH LeibnizInstitut für Höchstfrequenztechnik, 12489 Berlin, Germany; Helmholtz-Zentrum Berlin für Materialien und Energie, 14109 Berlin, Germany

Sviatoslav Kovalchuk - Department of Physics, Freie Universität Berlin, 14195 Berlin, Germany; 이이이이 0000-0002-4817-1939

Katja Höflich - Ferdinand-Braun-Institut gGmbH LeibnizInstitut für Höchstfrequenztechnik, 12489 Berlin, Germany; Helmholtz-Zentrum Berlin für Materialien und Energie, 14109 Berlin, Germany; 이이.org/0000-0003-40882928

Complete contact information is available at: https://pubs.acs.org/10.1021/acs.nanolett.0c04986

\section{Author Contributions}

J.N.K. conceived the idea. Suspended graphene devices were fabricated by K.W., S.K., and J.N.K. He-FIB pattering procedures were developed and carried out by K.H. and V.D. at HZB Berlin. S.H. acquired and analyzed Raman spectroscopy data. Sample design and FEM-modeling was performed by J.N.K. with participation by K.W. J.N.K. and K.I.B. cowrote the paper with input from all authors. K.I.B. supervised the project. All authors discussed the results.

\section{Funding}

This work was supported by ERC Starting Grant 639739 and DFG TRR 227. V.D. and K.H. acknowledge financial support from DFG under Grant HO 5461/3-1. The He ion beam patterning was performed in the Corelab Correlative Microscopy and Spectroscopy at Helmholtz-Zentrum Berlin and within the framework of the EU COST action CA 19140 'FIT4NANO'.

\section{Notes}

The authors declare no competing financial interest.

\section{REFERENCES}

(1) Kushwaha, M. S.; Halevi, P.; Dobrzynski, L.; Djafari-Rouhani, B. Acoustic Band Structure of Periodic Elastic Composites. Phys. Rev. Lett. 1993, 71 (13), 2022-2025.

(2) Hopkins, P. E.; Reinke, C. M.; Su, M. F.; Olsson, R. H.; Shaner, E. A.; Leseman, Z. C.; Serrano, J. R.; Phinney, L. M.; El-Kady, I. Reduction in the Thermal Conductivity of Single Crystalline Silicon by Phononic Crystal Patterning. Nano Lett. 2011, 11 (1), 107-112.

(3) Maldovan, M. Sound and Heat Revolutions in Phononics. Nature 2013, 503, 209-217.

(4) Mousavi, S. H.; Khanikaev, A. B.; Wang, Z. Topologically Protected Elastic Waves in Phononic Metamaterials. Nat. Commun. 2015, 6 (1), 1-7.

(5) Balram, K. C.; Davanço, M. I.; Song, J. D.; Srinivasan, K. Coherent Coupling between Radiofrequency, Optical and Acoustic Waves in Piezo-Optomechanical Circuits. Nat. Photonics 2016, 10 (5), $346-352$.

(6) Olsson, R. H.; El-Kady, I. Microfabricated Phononic Crystal Devices and Applications. Meas. Sci. Technol. 2009, 20 (1), 012002.

(7) Khelif, A.; Choujaa, A.; Benchabane, S.; Djafari-Rouhani, B.; Laude, V. Guiding and Bending of Acoustic Waves in Highly Confined Phononic Crystal Waveguides. Appl. Phys. Lett. 2004, 84 (22), 4400-4402.

(8) Lin, S. C. S.; Huang, T. J.; Sun, J. H.; Wu, T. T. Gradient-Index Phononic Crystals. Phys. Rev. B: Condens. Matter Mater. Phys. 2009, 79 (9), 094302.

(9) Zhao, J.; Bonello, B.; Marchal, R.; Boyko, O. Beam Path and Focusing of Flexural Lamb Waves within Phononic Crystal-Based Acoustic Lenses. New J. Phys. 2014, 16, 063031. 
(10) Benchabane, S.; Khelif, A.; Rauch, J. Y.; Robert, L.; Laude, V. Evidence for Complete Surface Wave Band Gap in a Piezoelectric Phononic Crystal. Phys. Rev. E - Stat. Nonlinear, Soft Matter Phys. 2006, 73 (6), 065601.

(11) Khelif, A.; Aoubiza, B.; Mohammadi, S.; Adibi, A.; Laude, V. Complete Band Gaps in Two-Dimensional Phononic Crystal Slabs. Phys. Rev. E - Stat. Nonlinear, Soft Matter Phys. 2006, 74 (4), 043509.

(12) Mohammadi, S.; Eftekhar, A. A.; Khelif, A.; Hunt, W. D.; Adibi, A. Evidence of Large High Frequency Complete Phononic Band Gaps in Silicon Phononic Crystal Plates. Appl. Phys. Lett. 2008, 92 (22), 221905.

(13) Li, F.; Liu, J.; Wu, Y. The Investigation of Point Defect Modes of Phononic Crystal for High Q Resonance. J. Appl. Phys. 2011, 109 (12), 124907.

(14) Yu, P. L.; Cicak, K.; Kampel, N. S.; Tsaturyan, Y.; Purdy, T. P.; Simmonds, R. W.; Regal, C. A. A Phononic Bandgap Shield for HighQ Membrane Microresonators. Appl. Phys. Lett. 2014, 104 (2), 023510 .

(15) Tsaturyan, Y.; Barg, A.; Polzik, E. S.; Schliesser, A. Ultracoherent Nanomechanical Resonators via Soft Clamping and Dissipation Dilution. Nat. Nanotechnol. 2017, 12 (8), 776-783.

(16) Ghadimi, A. H.; Wilson, D. J.; Kippenberg, T. J. Radiation and Internal Loss Engineering of High-Stress Silicon Nitride Nanobeams. Nano Lett. 2017, 17 (6), 3501-3505.

(17) Ghadimi, A. H.; Fedorov, S. A.; Engelsen, N. J.; Bereyhi, M. J.; Schilling, R.; Wilson, D. J.; Kippenberg, T. J. Elastic Strain Engineering for Ultralow Mechanical Dissipation. Science (Washington, DC, U. S.) 2018, 360 (6390), 764-768.

(18) Imboden, M.; Mohanty, P. Dissipation in Nanoelectromechanical Systems. Phys. Rep. 2014, 534, 89-146.

(19) Ekinci, K. L.; Roukes, M. L. Nanoelectromechanical Systems. Rev. Sci. Instrum. 2005, 76 (6), 061101.

(20) Guo, J.; Norte, R.; Gröblacher, S. Feedback Cooling of a Room Temperature Mechanical Oscillator Close to Its Motional Ground State. Phys. Rev. Lett. 2019, 123 (22), 223602.

(21) Delić, U.; Reisenbauer, M.; Dare, K.; Grass, D.; Vuletić, V.; Kiesel, N.; Aspelmeyer, M. Cooling of a Levitated Nanoparticle to the Motional Quantum Ground State. Science (Washington, DC, U. S.) 2020, 367 (6480), 892-895.

(22) Huang, H.; Tan, Z.; Huo, S.; Feng, L.; Chen, J.; Han, X. Topologically Protected Zero Refraction of Elastic Waves in Pseudospin-Hall Phononic Crystals. Commun. Phys. 2020, 3 (1), 46.

(23) Tian, Z.; Shen, C.; Li, J.; Reit, E.; Bachman, H.; Socolar, J. E. S.; Cummer, S. A.; Jun Huang, T. Dispersion Tuning and Route Reconfiguration of Acoustic Waves in Valley Topological Phononic Crystals. Nat. Commun. 2020, 11 (1), 762.

(24) Cha, J.; Daraio, C. Electrical Tuning of Elastic Wave Propagation in Nanomechanical Lattices at $\mathrm{MHz}$ Frequencies. Nat. Nanotechnol. 2018, 13, 1016-1020.

(25) Jang, J. H.; Ullal, C. K.; Gorishnyy, T.; Tsukruk, V. V.; Thomas, E. L. Mechanically Tunable Three-Dimensional Elastomeric Network/Air Structures via Interference Lithography. Nano Lett. 2006, 6 (4), 740-743.

(26) Lin, S. C. S.; Huang, T. J. Tunable Phononic Crystals with Anisotropic Inclusions. Phys. Rev. B: Condens. Matter Mater. Phys. 2011, 83 (17), 174303.

(27) Chen, Z. G.; Wu, Y. Tunable Topological Phononic Crystals. Phys. Rev. Appl. 2016, 5 (5), 054021.

(28) Lin, S. C. S.; Huang, T. J. Tunable Phononic Crystals with Anisotropic Inclusions. Phys. Rev. B: Condens. Matter Mater. Phys. 2011, 83 (17), 174303.

(29) Bian, Z.; Peng, W.; Song, J. Thermal Tuning of Band Structures in a One-Dimensional Phononic Crystal. J. Appl. Mech. 2014, 81 (4), 041008 .

(30) Jim, K. L.; Leung, C. W.; Lau, S. T.; Choy, S. H.; Chan, H. L. W. Thermal Tuning of Phononic Bandstructure in Ferroelectric Ceramic/Epoxy Phononic Crystal. Appl. Phys. Lett. 2009, 94 (19), 193501.
(31) Robillard, J. F.; Matar, O. B.; Vasseur, J. O.; Deymier, P. A.; Stippinger, M.; Hladky-Hennion, A. C.; Pennec, Y.; Djafari-Rouhani, B. Tunable Magnetoelastic Phononic Crystals. Appl. Phys. Lett. 2009, 95 (12), 124104.

(32) Bou Matar, O.; Robillard, J. F.; Vasseur, J. O.; Hladky-Hennion, A. C.; Deymier, P. A.; Pernod, P.; Preobrazhensky, V. Band Gap Tunability of Magneto-Elastic Phononic Crystal. J. Appl. Phys. 2012, 111 (5), 054901.

(33) Huang, Y.; Zhang, C. L.; Chen, W. Q. Tuning Band Structures of Two-Dimensional Phononic Crystals with Biasing Fields. J. Appl. Mech. 2014, 81 (9), 1-8.

(34) Thomas, R. A.; Parniak, M.; Østfeldt, C.; Møller, C. B.; Bærentsen, C.; Tsaturyan, Y.; Schliesser, A.; Appel, J.; Zeuthen, E.; Polzik, E. S. Entanglement between Distant Macroscopic Mechanical and Spin Systems. Nat. Phys. 2020, 1-6.

(35) Hatanaka, D.; Bachtold, A.; Yamaguchi, H. Electrostatically Induced Phononic Crystal. Phys. Rev. Appl. 2019, 11 (2), 1.

(36) Midtvedt, D.; Isacsson, A.; Croy, A. Nonlinear Phononics Using Atomically Thin Membranes. Nat. Commun. 2014, 5, 5.

(37) Wang, Y.; Lee, J.; Zheng, X. Q.; Xie, Y.; Feng, P. X. L. Hexagonal Boron Nitride Phononic Crystal Waveguides. ACS Photonics 2019, 6 (12), 3225-3232.

(38) Lee, C.; Wei, X.; Kysar, J. W.; Hone, J. Measurement of the Elastic Properties and Intrinsic Strength of Monolayer Graphene. Science (Washington, DC, U. S.) 2008, 321 (5887), 385-388.

(39) Nicholl, R. J. T.; Conley, H. J.; Lavrik, N. V.; Vlassiouk, I.; Puzyrev, Y. S.; Sreenivas, V. P.; Pantelides, S. T.; Bolotin, K. I. The Effect of Intrinsic Crumpling on the Mechanics of Free-Standing Graphene. Nat. Commun. 2015, 6, 8789.

(40) Singh, R.; Nicholl, R. J. T.; Bolotin, K. I.; Ghosh, S. Motion Transduction with Thermo-Mechanically Squeezed Graphene Resonator Modes. Nano Lett. 2018, 18 (11), 6719-6724.

(41) Bolotin, K. I.; Sikes, K. J.; Jiang, Z.; Klima, M.; Fudenberg, G.; Hone, J.; Kim, P.; Stormer, H. L. Ultrahigh Electron Mobility in Suspended Graphene. Solid State Commun. 2008, 146 (9-10), 351355.

(42) Deinhart, V. fib-o-mat. https://pypi.org/project/fibomat/ (accessed Oct 10, 2020).

(43) Lemme, M. C.; Bell, D. C.; Williams, J. R.; Stern, L. A.; Baugher, B. W. H.; Jarillo-Herrero, P.; Marcus, C. M. Etching of Graphene Devices with a Helium Ion Beam. ACS Nano 2009, 3 (9), 2674-2676.

(44) Archanjo, B. S.; Fragneaud, B.; Gustavo Cançado, L.; Winston, D.; Miao, F.; Alberto Achete, C.; Medeiros-Ribeiro, G. Graphene Nanoribbon Superlattices Fabricated via He Ion Lithography. Appl. Phys. Lett. 2014, 104 (19), 193114.

(45) Fox, D.; Zhou, Y. B.; O’Neill, A.; Kumar, S.; Wang, J. J.; Coleman, J. N.; Duesberg, G. S.; Donegan, J. F.; Zhang, H. Z. Helium Ion Microscopy of Graphene: Beam Damage, Image Quality and Edge Contrast. Nanotechnology 2013, 24 (33), 335702.

(46) Mohammadi, S.; Eftekhar, A. A.; Khelif, A.; Moubchir, H.; Westafer, R.; Hunt, W. D.; Adibi, A. Complete Phononic Bandgaps and Bandgap Maps in Two-Dimensional Silicon Phononic Crystal Plates. Electron. Lett. 2007, 43 (16), 898-899.

(47) Capelle, T.; Tsaturyan, Y.; Barg, A.; Schliesser, A. Polarimetric Analysis of Stress Anisotropy in Nanomechanical Silicon Nitride Resonators. Appl. Phys. Lett. 2017, 110 (18), 181106.

(48) Mohiuddin, T. M. G.; Lombardo, A.; Nair, R. R.; Bonetti, A.; Savini, G.; Jalil, R.; Bonini, N.; Basko, D. M.; Galiotis, C.; Marzari, N.; Novoselov, K. S.; Geim, A. K.; Ferrari, A. C. Uniaxial Strain in Graphene by Raman Spectroscopy: G Peak Splitting, Grüneisen Parameters, and Sample Orientation. Phys. Rev. B: Condens. Matter Mater. Phys. 2009, 79 (20), 1-8.

(49) Mueller, N. S.; Heeg, S.; Alvarez, M. P.; Kusch, P.; Wasserroth, S.; Clark, N.; Schedin, F.; Parthenios, J.; Papagelis, K.; Galiotis, C.; Kalbáč, M.; Vijayaraghavan, A.; Huebner, U.; Gorbachev, R.; Frank, O.; Reich, S. Evaluating Arbitrary Strain Configurations and Doping in Graphene with Raman Spectroscopy. 2D Mater. 2018, 5 (1), 015016. 
(50) Chen, C.; Rosenblatt, S.; Bolotin, K. I.; Kalb, W.; Kim, P.; Kymissis, I.; Stormer, H. L.; Heinz, T. F.; Hone, J. Performance of Monolayer Graphene Nanomechanical Resonators with Electrical Readout. Nat. Nanotechnol. 2009, 4 (12), 861-867.

(51) Tsaturyan, Y. Ultracoherent Soft-Clamped Mechanical Resonators for Quantum Cavity Optomechanics; University of Copenhagen, 2019. https://www.nbi.ku.dk/english/theses/phd-theses/yeghishetsaturyan/Yeghishe2.pdf.

(52) Alemán, B.; Regan, W.; Aloni, S.; Altoe, V.; Alem, N.; Girit, C.; Geng, B.; Maserati, L.; Crommie, M.; Wang, F.; Zettl, A. TransferFree Batch Fabrication of Large- Area Suspended Graphene Membranes. ACS Nano 2010, 4 (8), 4762-4768.

(53) Van Der Zande, A. M.; Barton, R. A.; Alden, J. S.; Ruiz-Vargas, C. S.; Whitney, W. S.; Pham, P. H. Q.; Park, J.; Parpia, J. M.; Craighead, H. G.; McEuen, P. L. Large-Scale Arrays of Single-Layer Graphene Resonators. Nano Lett. 2010, 10 (12), 4869-4873.

(54) Bunch, J. S.; Van Der Zande, A. M.; Verbridge, S. S.; Frank, I. W.; Tanenbaum, D. M.; Parpia, J. M.; Craighead, H. G.; McEuen, P. L. Electromechanical Resonators from Graphene Sheets. Science (Washington, DC, U. S.) 2007, 315 (5811), 490-493.

(55) Hauer, B. D.; Doolin, C.; Beach, K. S. D.; Davis, J. P. A General Procedure for Thermomechanical Calibration of Nano/MicroMechanical Resonators. Annals of Physics. Academic Press, 2013; pp 181-207.

(56) Waitz, R.; Nößner, S.; Hertkorn, M.; Schecker, O.; Scheer, E. Mode Shape and Dispersion Relation of Bending Waves in Thin Silicon Membranes. Phys. Rev. B: Condens. Matter Mater. Phys. 2012, 85 (3), 035324

(57) Chen, C.; Lee, S.; Deshpande, V. V.; Lee, G. H.; Lekas, M.; Shepard, K.; Hone, J. Graphene Mechanical Oscillators with Tunable Frequency. Nat. Nanotechnol. 2013, 8 (12), 923-927. 